Revista Chilena
DE Pediatría

www.revistachilenadepediatria.cl

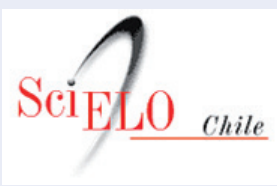

www.scielo.cl

Rev Chil Pediatr. 2020;91(6):999

DOI: $10.32641 /$ rchped.v91i6.3480

CARTA AL EDITOR

\section{Antibióticos en neonatología}

\section{Antibiotics in neonatology}

\section{Sr. Editor,}

Los antimicrobianos son los medicamentos más utilizados en unidades de neonatología durante el primer mes de vida principalmente porque esta población presenta alto riesgo de adquirir infecciones graves y rapidamente progresivas hacia un descenlace fatal como es la sepsis ${ }^{1,2}$.

Lamentablemente en forma histórica la mayoría de las dosificaciones de los antimicrobianos utilizados en esta población se basan en extrapolaciones de estudios en población adulta y pediátrica, pese a que la fisiopatología de los recién nacidos difiere bastante de estos dos grupos y a que, por sobre estos cambios, influyen variables extras como son aquellos ocasionados por los amplios volúmenes de distribución como ocurre en el shock séptico por ejemplo ${ }^{3}$. El hecho de utilizar dosis no diseñadas para la población de neonatos lleva a un mayor riesgo de efectos adversos los que pueden conducir a una mayor toxicidad, resistencia bacteriana y a fallas terapéuticas ${ }^{4}$.

La adecuada y certera dosificación de antibióticos en recién nacidos ha sido un desafío permanente debido a la escasez de datos confiables disponibles, producto de: preocupaciones éticas para la incorporación de neonatos en estudios experimentales, escasa disponibilidad de pacientes, falta de diseño adecuado de los estudios clínicos realizados, falta de experiencia en simulación farmacocinética/farmacodinámica neonatal y grandes volúmenes de sangre requeridos para los análisis de medición de niveles plasmáticos.

Gracias a que en la última década se han realizado mayores estudios con enfoques farmacocinéticos y farmacodinámicos en neonatos, hoy en día podemos contar con nueva evidencia y de mejor calidad sobre la dosificación de la mayoría de los antimicrobianos más utilizados, y es por ello que recientemente se elaboró y publicó el documento: “Antimicrobianos en neonato- logía. Parte I: Recomendaciones de dosificaciones basadas, en la más reciente evidencia en recién nacidos. Comité Consultivo de Infecciones Neonatales, Sociedad Chilena de Infectología", con la idea de entregar una mejor herramienta técnica, resumida y actualizada y así facilitar y promover el correcto uso de antimicrobianos en las unidades neonatales ${ }^{5}$.

El documento contiene una puesta al día de 16 antimicrobianos, con sus respectivas tablas de recomendación de dosis y un pequeño resumen de cada uno de los antibióticos mencionados ${ }^{5}$, con la idea de apoyar de esta forma una adecuada gestión de antibióticos a las unidades neonatales.

\section{Alejandra Sandoval Carmona $a^{a, b, c}$ \\ aPediatra Infectóloga, Hospital Carlos Van Buren. Valparaíso, Chile. \\ ${ }^{b}$ Departamento de Pediatría, Escuela de Medicina, Universidad de Valparaíso. Valparaíso, Chile. ${ }^{c}$ Comité Consultivo de Infecciones Neonatales, Sociedad Chilena de Infectología. Santiago, Chile.}

\section{Referencias}

1. Rivera-Chaparro ND, Cohen-Wolkowiez M, Greenberg RG. Dosing antibiotics in neonates: review of the pharmacokinetic data. Future Microbiol. 2017;12: 1001-16. doi: 10.2217/fmb2017-0058.

2. van Donge T, Bielicki JA, van den Anker J, Pfister M. Key Components for antibiotic dose optimization of sepsis in neonates and infants. Front Pediatr. 2018;6:325. doi: 10.3389/ fped.2018.00325.

3. Cohen-Wolkowiez M, Watt KM, Zhou C, et al. Developmental pharmacokinetics of piperacillin and tazobactam using plasma and dried blood spots from infants. Antimicrob Agents Chemother. 2014;58(5):2856-65. doi: 10.1128/AAC.02139-13.

4. Thompson G, Barker CI, Folgori L, et al. Global shortage of neonatal and paediatric antibiotic trials: rapid review. BMJ Open. 2017;7(10):e016293. doi: 10.1136/ bmjopen-2017-016293.

5. Sandoval A, Aravena M, Cofré F, et al. Antimicrobianos en neonatología. Parte I: Recomendaciones de dosificaciones basadas, en la más reciente evidencia en recién nacidos Comité Consultivo de Infecciones Neonatales, Sociedad Chilena de Infectología. Rev Chilena Infectol 2020;37(5):561-79.

Correspondencia: 